\title{
Estudio de la patología endometrial con base en la biopsia de endometrio
}

\author{
M. Rebolledo MD.*; F. Arbeláez MD.**; A.M. Nariño MD.***
}

\begin{abstract}
RESUMEN: En el Hospital Infantil Universitario "Lorencita Villegas de Santos” el raspado fraccionado es el método diagnóstico usual para el estudio de la patología endometrial. Teniendo en cuenta que la biopsia es una herramienta confiable, sencilla, económica y segura para la paciente, se decidió establecer la confiabilidad de ésta para evaluar la patología endometrial. Para cumplir ese objetivo se estudiaron 22 pacientes mayores de 35 años programadas para histerectomía abdominal a las que se les tomó una biopsia de endometrio con anterioridad a la cirugía. Se compararon los resultados del estudio histopatológico de las biopsias con el reporte del endometrio de la pieza quirúrgica. En todas las pacientes se obtuvo material para estudio, que fue adecuado para dar un diagnóstico en 19 pacientes $(86.3 \%)$ e insuficiente en $3(13.6 \%)$. La correlación del resultado de la biopsia frente al endometrio de la pieza quirúrgica fue del $100 \%$. Con base en estos resultados consideramos que la biopsia es una alternativa adecuada para el estudio de las pacientes con hemorragia uterina anormal, conociendo adecuadamente sus limitaciones y acudiendo al raspado fraccionado cuando con la biopsia no se logra un diagnóstico adecuado.
\end{abstract}

PALABRAS CLAVES: Hemorragia uterina anormal, biopsia de endometrio, raspado fraccionado, patología.

SUMMARY: Dilatation and curettage is the usual diagnostic method for the study of endometrial pathology at Hospital Infantil Universitario "Lorencita Villegas de Santos". Having in mind that biopsy is a trustful, easy, economic and safe tool for the patient, we decided to stablish its degree of confiability in the evaluation of endometrial pathology.

To acomplish this objetive, a biopsy was preformed prior to surgery in 22 patients over 35 years of age, that were programed for total abdominal histerectomy. The pathology results of the biopsy were compared with those of the endometrium in the surgical piece. In all patients material was obtained for study, wich was adecuate for diagnosis in 19 patients $(86.3 \%)$ and insufficient in 3 (13.6\%). The correlation between the biopsy and the endometrium in the surgical sample was $100 \%$.

Taking these results on account, we consider that biopsy is an adecuate alternative for the study of patients with abnormal uterine bleeding, as long as its limitations are known and dilatation and curettage is used whenever it is not possible to obtain an adecuate diagnosis with the biopsy.

KEY WORDS: Abnormal uterine bleeding, biopsy of endometrium, dilatation and curettage, pathology.

\section{Introducción}

El raspado fraccionado es el método estándar para el diagnóstico de la patología endometrial, convirtiéndose en el procedimiento ginecológico hospitalario más común en los Estados Unidos con 997.000 anuales (1-4). Su sensibilidad y especificidad son superiores al $95 \%$, sin embargo presenta desventajas como la necesidad de anestesia y hospitalización, lo que se refleja en aumento de los costos, de la misma manera que en riesgos inherentes de morbimortalidad, además de la frecuente incomodidad para la paciente (2-3).

Lo anterior ha llevado a tener en cuenta unos procedimientos menos invasivos y costosos, como herramientas diagnósticas para la patología endometrial entre los que encontramos la citología cervicovaginal, el lavado y la biopsia endometrial (1-8). Los dos primeros tienen una sensibilidad menor del $50 \%$ con una baja especificidad

* Residente III Departamento de Ginecología y Obstetricia Hospital Infantil Universitario "Lorencita Villegas de Santos".

** Instructor Departamento de Ginecología y Obstetricia Hospital Infantil Universitario "Lorencita Villegas de Santos".

*** Especialista en Patología Universidad del Rosario.
(5, 9-14). La Biopsia Endometrial es un procedimiento ambulatorio, de bajo costo, que no requiere anestesia (2, $5,15-16)$. Ha sido evaluado por varios grupos de investigadores encontrando una fácil interpretación, con sensibilidad, especificidad, al igual que la calidad de muestreo histológico satisfactorio, semejante al raspado fraccionado $(9,16)$. El material obtenido por biopsia es comparable al de la dilatación y curetaje $(5-6,9,17)$.

En el Hospital Infantil Universitario Lorencita Villegas de Santos se utiliza actualmente en forma rutinaria el raspado fraccionado como método de elección para el estudio de la patología del endometrio en las pacientes mayores de 35 años. Teniendo en cuenta que la biopsia de endometrio es un procedimiento sencillo, económico y seguro para la paciente, y que en nuestra institución no hay antecedentes de un estudio en el cual se valore esta herramienta diagnóstica se decidió establecer la confiabilidad de la biopsia para el diagnóstico de patología endometrial, de manera tal que este método reemplace al raspado fraccionado como método diagnóstico inicial de la hemorragia uterina anormal en las pacientes que no tienen factores de riesgo para el desarrollo de carcinoma de endometrio. 
Para cumplir este objetivo se intentó demostrar que el material histológico obtenido a través de la biopsia endometrial era representativo y confiable para el diagnóstico histopatológico, al compararlo con el resultado obtenido en una pieza quirúrgica.

\section{Materiales y métodos}

Se realizó un trabajo prospectivo y descriptivo no randomizado.

Se estudiaron las pacientes que asistieron a los servicios de urgencias y consulta externa de Ginecología en el Hospital Infantil Universitario "Lorencita Villegas de Santos", entre el 1o. de enero de 1994 y el 31 de octubre del mismo año (10 meses de estudio), que tuvieran 35 años de edad o más que consultaron por presentar hemorragia uterina anormal. Posterior a un estudio detallado de su patología se decidió programar para la realización de histerectomía abdominal.

Se les tomó biopsia de endometrio a todas las pacientes programadas para histerectomía abdominal por cualquier indicación, excluyendo las que requerían una histerectomía de urgencia de las pacientes con carcinoma de cérvix, y las que no aceptaron el procedimiento. A todas se les realizó la valoración clínica y paraclínica acostumbrada en el hospital, pertinente a su patología, en los formatos de historia ginecológica, de donde se obtuvieron los datos clínicos.

La biopsia se realizó en forma ambulatoria en la última consulta previa a la cirugía, o el día anterior a ésta en el momento de ingreso a la institución.

En todos los casos se obtuvieron los siguientes datos: antecedentes gineco-obstétricos, familiares, y personales, factores de riesgo para carcinoma de endometrio, métodos de planificación familiar, resultados de citologías cervicovaginales previas, motivo de consulta y descripción de la enfermedad actual, diagnóstico, e indicación de la histerectomía.

El procedimiento se hizo de la siguiente manera, previo diligenciamiento de un formato de aceptación y consentimiento del procedimiento (18-19): Un tacto vaginal para establecer la orientación uterina, y las características de los genitales internos, luego cervicovaginoscopia con asepsia de los fondos de saco y el cuello uterino, pinzamiento del labio anterior con el tenáculo de Jacobs, y posteriormente toma de la biopsia endometrial de las 2 caras y los dos bordes uterinos con la cureta de Novak, según se describió previamente. El material obtenido se fijó con formaldehído al $10 \%$ inmediatamente después. Las muestras de las biopsias de endometrio fueron procesadas y leídas por uno de los investigadores, quien no conocía los datos clínicos de la paciente que consignó los hallazgos macroscópicos y microscópicos, el diagnóstico histopatológico y una calificación de la calidad de la muestra para su lectura como adecuada o inadecuada.

Posteriormente, se obtuvo el reporte de patología del endometrio en el espécimen quirúrgico, procesado en el laboratorio de patología del hospital.

Los resultados histopatológicos para su comparación se clasificaron de la siguiente forma:
1. Endometrio cíclico, que puede ser proliferativo, secretor o menstrual.

2. Endometrio con hiperplasia, simple, compleja, con atipias o sin atipias.

3. Carcinoma de endometrio.

4. Endometrio atrófico.

5. Endometrio con efecto farmacológico exógeno.

6. Otros.

Los resultados de calidad de la muestra y de la concordancia histopatológica se analizan anotando las características descritas por el patólogo y se expresan en porcentaje del número total de pacientes estudiadas.

\section{Resultados}

De las 22 pacientes analizadas durante los 10 meses la distribución de acuerdo con los grupos de edad fue la siguiente: Entre 35 y 45 años 12 pacientes (54.5\%), y entre 46 y 55 años $10(45.4 \%)$ lo cual se expresa en la tabla 1.

Al revisar los antecedentes familiares se encontró datos positivos en 9 de ellas (40.9\%), siendo el más frecuente la hipertensión arterial en familiares de primer grado de consanguinidad en 7 pacientes $(31.8 \%)$, y diabetes mellitus no insulino dependientes en 2 pacientes (9\%). Ver tabla 2.

Se observaron antecedentes personales asociados al desarrollo de carcinoma endometrial en 5 de éstas (22.7\%), manifestando la concomitancia de dos patologías específicamente obesidad e hipertensión en 2 pacientes $(9 \%)$, e hipertensión y obesidad aisladas en otras 2 pacientes $(9 \%)$. Sólo una paciente tenía el antecedente de diabetes asociado a obesidad (4.5\%). Ver tabla 3.

Todas las pacientes tenían citologías cervicovaginales negativas para neoplasia.

El motivo de consulta de 21 de las 22 pacientes (95.4\%) fueron las alteraciones menstruales de las cuales las más frecuentes eran polihipermenorreas en $16(72.7 \%)$ y metrorragias en $4(18.1 \%)$. En una paciente se encontró el antecedente de oligomenorreas $(4.5 \%)$ y otra había consultado por dolor pélvico (4.5\%). Ver tabla. 4.

El tiempo de evolución de la sintomatología estaba entre 4 meses y 8 años. En 7 pacientes fue menor de un año (31.8\%), y en el resto de ellas era mayor de 1 año (15 pacientes para el $68.1 \%$. Ver tabla 5).

En lo que respecta a la biopsia endometrial, el reporte del patólogo de la calidad de ésta, obtenida con cureta de Novak, es señalada como adecuado en 19 pacientes (86.3\%), e inadecuado por ser insuficiente para el diagnóstico histopatológico, en $3(13.6 \%)$. Ver tabla 6.

De la misma manera 17 pacientes tenían endometrio cíclico $(77.2 \%)$ de los cuales era proliferativo en 12 pacientes $(54.5 \%)$, secretor en $4(18.1 \%)$ y uno descamativo $(4.5 \%)$. En una paciente se obtuvo endometrio con efecto farmacológico exógeno de tipo progestacional $(4.5 \%)$ y en otra un endometrio atrófico $(4.5 \%)$. Además tres pacientes evidenciaban una patología adicional, dos de las cuales eran focos aislados de hiperplasia simple y en otra un pólipo endometrial $(13.6 \%)$. Ver tabla 7.

Los resultados de patología en la pieza quirúrgica mostraron un endometrio cíclico en 20 pacientes (90.9\%), 
Tabla 1

DISTRIBUCION DE LA EDAD

Distribución de las pacientes según grupos etáreos de edad

\begin{tabular}{|ccc|}
\hline Edad & No. Pacientes & $\%$ \\
\hline $35-45$ & 12 & 54.5 \\
$46-55$ & 10 & 45.4 \\
\hline
\end{tabular}

Promedio: 43 Años

Tabla 2

ANTECEDENTES FAMILIARES

Pacientes con antecedentes familiares de hipertensión y diabetes

\begin{tabular}{|lcc|}
\hline Antecedente & No. Pacientes & $\%$ \\
\hline Hipertensión & 7 & 31.8 \\
Diabetes & 2 & 9 \\
\hline
\end{tabular}

Total \% pacientes con antecedentes familiares $36.3 \%$.

Tabla 3

ANTECEDENTES PERSONALES

Pacientes con antecedentes personales de hipertensión arterial, obesidad o diabetes no insulino dependiente

\begin{tabular}{|lll|}
\hline Antecedente & No. Pacientes & \% \\
\hline Hipertensión & 1 & 4.5 \\
Obesidad & 1 & 4.5 \\
Hipertensión-Obesidad & 2 & 9 \\
Diabetes-Obesidad & 1 & 4.5 \\
\hline
\end{tabular}

$\%$ Total de pacientes con antecedentes personales asociados con aumento de riesgo para carcinoma de endometrio $22.7 \%$.

Tabla 4

TIPO DE SINTOMATOLOGIA

Sintomatología que llevó al inicio del estudio clínico que finalmente termino en la decisión de histerectomía abdominal

\begin{tabular}{|c|c|c|}
\hline Síntoma & $\%$ Pacientes & $\%$ \\
\hline $\begin{array}{l}\text { Hemorragia Uterina } \\
\text { Anormal }\end{array}$ & 21 & 95.4 \\
\hline Polihipermenorreas & 16 & 72.7 \\
\hline Metrorragia & 4 & 18.1 \\
\hline Oligomenorreas & 1 & 4.5 \\
\hline Dolor pélvico & 1 & 4.5 \\
\hline
\end{tabular}

Tabla 5

TIEMPO DE EVOLUCION

Tiempo de evolución de la sintomatología hasta el momento de la primera consulta

\begin{tabular}{|ccc|}
\hline Tiempo & No. Pacientes & $\%$ \\
\hline$<1$ año & 7 & 31.8 \\
$>1$ año & 15 & 68.1 \\
\hline
\end{tabular}

Promedio 18 meses (4-60 meses)

\section{Tabla 6}

CALIDAD DE LA BIOPSIA DE ENDOMETRIO

Calificación de la calidad de la muestra para el estudio histopatológico. Es inadecuada cuando el material es insuficiente para un diagnóstico

\begin{tabular}{|ccc|}
\hline No. Pacientes & Calificación & \% \\
\hline 19 & Adecuada & 86.3 \\
3 & Inadecuada & 13.6 \\
\hline
\end{tabular}

Tabla 7

RESULTADOS BIOPSIA ENDOMETRIAL

Clasificación de los diagnósticos histopatológicos obtenidos en las biopsias de endometrio

\begin{tabular}{|lcc|}
\hline Diagnóstico & No. Pacientes & \% \\
\hline Cíclico & 17 & 77.2 \\
Proliferativo & 11 & 50 \\
Secretor & 5 & 22.7 \\
Descamativo & 1 & 4.5 \\
Atrófico & 1 & 4.5 \\
Acción hormonal exógena & 1 & 4.5 \\
Material insuficiente & 3 & 13.6 \\
\hline
\end{tabular}

Tabla 8

\section{RESULTADOS ENDOMETRIO PIEZA QUIRURGICA}

Clasificación de los diagnósticos histopatológicos del endometrio de las piezas quirúrgicas

\begin{tabular}{|lcc|}
\hline Diagnóstico & No. pacientes & $\%$ \\
\hline Cíclico & 20 & 90.9 \\
Proliferativo & 13 & 59 \\
Secretor & 7 & 31.8 \\
Descamativo & & \\
Atrófico & 1 & 4.5 \\
Acción hormonal exógena & 1 & 4.5 \\
\hline
\end{tabular}

una paciente con endometrio atrófico $(4.5 \%)$ y otra con terapia hormonal exógena de tipo progestacional $(4.5 \%)$. En una paciente se encontró además un pólipo endometrial $(4.5 \%)$. Ver tabla 8.

Para analizar la concordancia de los resultados se clasificaron en 6 grupos como se mencionó en los materiales y métodos: Endometrio cíclico, hiperplásico, carcinoma de endometrio, endometrio atrófico, con efecto farmacológico exógeno y otros. En la tabla 9 se presentan los resultados de las 22 pacientes en una forma comparativa del material obtenido en la biopsia frente al de la pieza quirúrgica incluyendo las pacientes con material inadecuado. Se observó una correlación del 100\%. 
Tabla 9

RESULTADOS BIOPSIA DE ENDOMETRIO VS ENDOMETRIO PIEZA QUIRURGICA

Tabla comparativa de los resultados del estudio patológico de la biopsia endometrial vs endometrio de la pieza quirúrgica paciente por paciente

\begin{tabular}{|lll|}
\hline & Biopsia endometrio & Endometrio pieza quirúrgica \\
\hline 1 & Cíclico (proliferativo) & Cíclico (proliferativo) \\
2 & Cíclico (secretor) & Cíclico (proliferativo) \\
3 & Cíclico (proliferativo-focos hiperplasia quística) & Cíclico (proliferativo) \\
4 & Atrófico & Atrófico \\
5 & Cíclico (proliferativo) & Cíclico (proliferativo) \\
6 & Cíclico (proliferativo) & Cíclico (proliferativo) \\
7 & Cíclico (proliferativo) & Cíclico (proliferativo) \\
8 & Material Insuficiente & Cíclico (secretor) \\
9 & Cíclico (proliferativo) & Cíclico (proliferativo) \\
10 & Cíclico (secretor) & Cíclico (secretor) \\
11 & Cíclico (secretor) & Cíclico (secretor) \\
12 & Acción hormonal exógena progestacional & Acción hormonal exógena progestacional \\
13 & Cíclico (proliferativo) & Cíclico (proliferativo) \\
14 & Material insuficiente & Cíclico (proliferativo) \\
15 & Cíclico (proliferativo-focos hiperplasia simple) & Cíclico (secretor-pólipo endometrial) \\
16 & Cíclico (descamativo) & Cíclico (proliferativo) \\
17 & Cíclico (proliferativo) & Cíclico (secretor) \\
18 & Material insuficiente & Cíclico (proliferativo) \\
19 & Cíclico (proliferativo-pólipo endometrial) & Cíclico (proliferativo) \\
20 & Cíclico (secretor) & Cíclico (secretor) \\
21 & Cíclico (proliferativo) & Cíclico (proliferativo) \\
22 & Cíclico (secretor) & Cíclico (secretor) \\
\hline
\end{tabular}

\section{Discusión}

En los países desarrollados, el carcinoma de endometrio es actualmente la enfermedad ginecológica maligna más frecuente, duplicando incluso, al carcinoma invasivo de cérvix (5-6), con una incidencia de 0.72 por 1.000 mujeres, 37.000 casos nuevos y 3.000 muertes cada año (7), sin embargo en nuestro país aún es la segunda causa de malignidad del tracto genital femenino (20).

El diagnóstico del carcinoma de cérvix es actualmente un procedimiento de consultorio (7) mientras que el de endometrio sigue siendo hospitalario requiriendo de un raspado fraccionado $(1,7,21)$.

El raspado fraccionado tiene una sensibilidad y especificidad superiores al 95\%, sin embargo presenta desventajas como la necesidad de anestesia y hospitalización, lo que refleja un aumento de los costos, y en los riesgos inherentes de morbimortalidad (2-4). Las complicaciones relacionadas con el procedimiento son hemorragia con una incidencia de 4 por 1.000 , infección en 4 a 5 por 1.000 , y perforación uterina en 3 a 13 por $1.000(2-4)$.

Esas dificultades asociadas a la Dilatación y Curetaje han llevado desde hace varios años a evaluar procedimientos sencillos, de consultorio para el estudio del endometrio de pacientes con hemorragia uterina anormal (6).

Entre estos métodos diagnósticos se menciona:
- La Citología cervicovaginal, que no es adecuada para valorar la hemorragia uterina, ya que sólo tiene una positividad del 35 al $80 \%$ y una tasa de falsos negativos del $82 \%$ para el diagnóstico de carcinoma de endometrio $(5,9-13)$.

- Los lavados endometriales, mediante varios dispositivos que permiten obtener células endometriales, sólo logran un $60 \%$ de muestras satisfactorias, siendo únicamente adecuados para el diagnóstico de casos avanzados de carcinoma de endometrio $(5,9,14)$.

- La Biopsia Endometrial, propuesta hace más de 50 años por Howard Kelly como "raspado sin anestesia en el consultorio" $(2,5,15-16)$, es un procedimiento ambulatorio, de bajo costo, que no requiere anestesia. Se han diseñado varios instrumentos para obtener las muestras, de los cuales el más aceptado y evaluado actualmente es la cureta de Novak $(4,22)$.

Diferentes grupos han evaluado este método y han encontrado lo siguiente: una fácil interpretación histológica, éxito en obtención de la muestra sin anestesia hasta en el $90 \%$ de los casos; aceptación por el paciente en el $90 \%$; un $91.7 \%$ de confiabilidad para detectar precursores de cáncer endometrial; un $97.5 \%$ de exactitud para detectar carcinoma de endometrio $(9,16)$. También se ha descrito que el material obtenido por biopsia 
es comparable al de la dilatación y curetaje $(5-6,9,17$, 23). En Colombia se ha evaluado este método diagnóstico, encontrando una correlación entre biopsia y raspado uterino de alrededor del 96\% (24-25).

En el Hospital Infantil Universitario "Lorencita Villegas de Santos" no hay antecedentes de una evaluación de este método diagnóstico, utilizándose al raspado fraccionado para la evaluación de la hemorragia uterina anormal en todos los casos.

En este estudio, en el que se incluyeron 22 pacientes, se pudo obtener una muestra sin anestesia en el $100 \%$ de ellas y fue un procedimiento aceptable para todas estas, aunque no se evaluó la intensidad del dolor referido por la paciente. El material obtenido fue adecuado para hacer un diagnóstico histopatológico en el $86.3 \%$ de las pacientes. Se obtuvo una muestra insuficiente en $3(13.6 \%)$ de las pacientes estudiadas, en las que sin embargo, no se encontró ninguna lesión maligna o premaligna en el endometrio de la pieza quirúrgica.

Es relevante que en tres de las pacientes estudiadas (13.6\% de las muestras) se encontraron elementos histopatológicos focales diferentes al tipo de endometrio de base (focos de hiperplasia simple y un pólipo endometrial).

En las 19 pacientes en las que el material de la biopsia fue adecuado para estudio se encontró una concordancia en todos con el diagnóstico histopatológico del endometrio de las piezas quirúrgicas obtenidas, lo que nos da un $100 \%$ de concordancia.

Las complicaciones relacionadas con este procedimiento son la perforación uterina, la enfermedad pélvica inflamatoria y la interrupción inadvertida de un embarazo temprano, que se presentan en un bajo porcentaje de los casos $(9,16)$. En ninguna de las pacientes estudiadas se presentó alguna complicación relacionada con la toma de la biopsia, aunque la muestra es muy pequeña para evaluar la frecuencia de las complicaciones asociadas a esta técnica diagnóstica. Está contraindicado en casos de falta de visualización del cérvix por procedimientos previos, colpoestenosis por radioterapia previa, senilidad, infección cervical o vaginal, no deseo de la paciente
(17-18, 26-27), antecedentes que no tenían ninguna de nuestras pacientes.

Con base en la concordancia obtenida entre la biopsia de endometrio y el endometrio de la pieza quirúrgica consideramos que la biopsia es una alternativa adecuada para el estudio endometrial de las pacientes con hemorragia uterina normal que no tengan factores de riesgo para el desarrollo de carcinoma de endometrio, siendo especialmente útil en las pacientes que van a ser sometidas a histerectomía por alguna patología no relacionada con el endometrio, en las que se debe tener un estudio del endometrio como parte de la evaluación prequirúrgica.

Una razonable aproximación para el manejo del sangrado anormal en mujeres mayores de 35 años es realizar directamente dilatación y curetaje en los casos en que hay factores de riesgo; cuando estos no existen, el procedimiento diagnóstico de elección es la biopsia endometrial; pero con una biopsia negativa y persistencia de los síntomas, sin una etiología aparente (v.gr. miomatosis uterina) el procedimiento adecuado es el raspado fraccionado. Cuando no hay síntomas y hay factores de riesgo, la biopsia endometrial es un procedimiento adecuado de seguimiento.

Actualmente algunos consideran que la biopsia puede ser el método diagnóstico inicial de elección excepto cuando se desee controlar una hemorragia activa. Se debe tener en cuenta que en algunas circunstancias el resultado de la biopsia debe ser seguido por un raspado, como en los casos de hiperplasia endometrial, a causa de la multifocalidad de las lesiones endometriales. Una muestra insuficiente no debe producir una falsa sensación de seguridad.

\section{Conclusiones}

La biopsia de endometrio es una alternativa segura, confiable y económica para el estudio de la patología endometrial en pacientes sin factores de riesgo para carcinoma de endometrio, especialmente útil en las pacientes que van a ser sometidas a histerectomía abdominal para tener un diagnóstico histopatológico del endometrio antes de la cirugía.

\section{BIBLIOGRAFIA}

1. Finkler NJ., Friedman AJ. The uterine corpus in Kenneth JR., Berkowitz R., Barbieri RL: Kistner's Gynecology: Principles and practice. Year Book Medical Publishers. Fifth edition 1990; 188.

2. Mattlingly RF., Thompson JD. Tumores malignos del útero en Ginecología Operatoria de Telinde. El Ateneo. Sexta edición 1987; 747.

3. Grimes DA. Diagnostic dilation and curettage: A reappraisal. Obstet. Gynecol. 1982; 142: 1 .

4. Smith JJ., Schulman H. Current dilatation and curettage practice: A need for revision. Obstet. Gynecol. 1985; 65: 516.

5. Hofmeister FJ. Endometrial biopsy: Another look. Am. J. Obstet. Gynecol. 1974; 118: 773.

6. Kohl GC., Larson PL. Office endometrial biopsies. Am. J. Obstet. Gynecol. 1974; 118: 406.

7. Disaia PJ., Creasman WT. Adenocarcinoma del útero en Disaia PJ., Creasman WT: Ginecología Oncológica. Editorial Médica Panamericana. Tercera edición 1991; 124.

8. Jones HW., Jones GS: Carcinoma endometrial en Tratado de Ginecología de Novak. Interamericana. Décima edición 1988; 394.
9. Koss LG., Schreiber K., Oberlander SG et al. Detection of endometrial and hyperplasia in asymptomatic women. Obstet. Gynecol. 1984; 64: 1 .

10. Anderson DG., Eaton CJ., Galinkin LJ et al. The cytologic diagnosis of endometrial adenocarcinoma. Am. J. Obstet. Gynecol. 1976; 125: 376 .

11. Burk JR., Lehman HF., Wolf FS. Inadequacy of papanicolaou smears in the detection of endometrial cancer. N. Eng. J. Med. 1974; 291: 191.

12. Isaacs JH., Ross FH. Cytologic evaluation of the endometrium in women with postmenopausal bleeding. Am. J. Obstet. Gynecol. 1978; 131: 410.

13. Zucker PK., Kasdon EJ., Feldstein ML. The validity of pap smear parameters as predictors of endometrial pathology in menopausal women. Cancer 1985; 56: 2.256.

14. Ellice RM., Morse AR., Anderson MC. Aspiration cytology versus histology in the assesment of the endometrium of women attending a menopause clinic. Br. J. Obstet. Gynaecol. 1981; 88: 421. 
15. Friedman, Barten Chapin. Gynecological Decision Making. B.C. Decker Inc. 1988; 92.

16. Gusberg SB., Milano C. Detection of endometrial cancer and its precursors. Cancer 1981; 47: 1173.

17. Greenwood SN., Wright J. Evaluation of the office endometrial biopsy in the detection of the endometrial carcinoma and atypical hyperplasia. Cancer 1979; 43: 1474.

18. Koss LG. Schreiber K., Oberlander SG. Screening of asymptomatic women for endometrial cancer. Obstet. Gynecol. 1981; 57: 681.

19. Mattlingly RF., Thompson LD. Dilatación del cuello y curetaje del útero en Ginecología Operatoria de Telinde. El Ateneo. Sexta edición 1987; 432.

20. Instituto Nacional de Cancerología. Asociación Colombiana de Ginecólogos Oncólogos: Manual de Normas de Manejo del Cáncer Genital Femenino. Rev. Col. Obstet. Ginecol. Suplemento. Marzo de 1993.

21. Anderson B. Diagnosis of endometrial cancer in Clinics in Obstetrics and Gynaecology. 1986; 13(4).
22. Silver MM., Miles P., Rosa C. Comparison of Novak and Pipelle endometrial biopsy instruments. Obstet. Gynecol. 1991; 78: 828.

23. Archer DF., McIntyre-Seltman K., Wilborn WW et al. Endometrial morphology in asymptomatic postmenopausal women. Am. J. Obstet. Gynecol. 1991; 165: 317.

24. Moreno-Escallón B., Angulo A., Botero LF et al. Manejo de la hiperplasia endometrial con acetato de medroxiprogesterona de depósito. Rev. Col. Obstet. Ginecol. 1991; 42: 63.

25. Toledo VA., Díaz CR. Evaluación de la biopsia versus legrado en diagnóstico de patología endometrial. Hospital Militar Central. Trabajo no publicado.

26. Koss LG., Schreiber K., Oberlander SG et al. Detection of endometrial carcinoma and hyperplasia in asymptomatic women. Obstet. Gynecol. 1984; 64: 1 .

27. Stovali TG., Ling FW., Morgan PL. A prospective, randomized comparison of the Pipelle endometrial sampling device with the Novak curette. Am. J. Obstet. Gynecol. 1991; 165: 1287. 